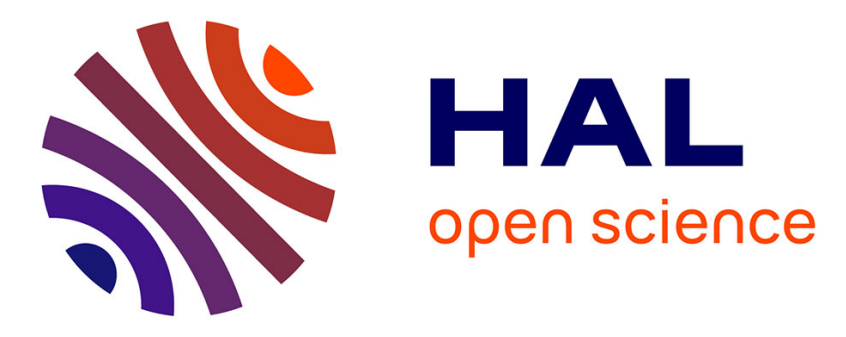

\title{
A family of functions with two different spectra of singularities
}

\author{
Claire Coiffard Marre, Clothilde Melot, Thomas Willer
}

\section{To cite this version:}

Claire Coiffard Marre, Clothilde Melot, Thomas Willer. A family of functions with two different spectra of singularities. Journal of Fourier Analysis and Applications, 2014, 20 (5), pp.961-984. 10.1007/s00041-014-9341-6 . hal-00831404v5

\section{HAL Id: hal-00831404 \\ https://hal.science/hal-00831404v5}

Submitted on 30 Jul 2015

HAL is a multi-disciplinary open access archive for the deposit and dissemination of scientific research documents, whether they are published or not. The documents may come from teaching and research institutions in France or abroad, or from public or private research centers.
L'archive ouverte pluridisciplinaire HAL, est destinée au dépôt et à la diffusion de documents scientifiques de niveau recherche, publiés ou non, émanant des établissements d'enseignement et de recherche français ou étrangers, des laboratoires publics ou privés. 


\title{
A family of functions with two different spectra of singularities
}

\author{
Claire Coiffard ${ }^{*}$ Clothilde Mélot † Thomas Willer $\ddagger$ \\ Aix-Marseille Université, CNRS, Centrale Marseille, I2M \\ UMR 7373, 13453 Marseille France
}

\begin{abstract}
Our goal is to study the multifractal properties of functions of a given family which have few non vanishing wavelet coefficients. We compute at each point the pointwise Hölder exponent of these functions and also their local $L^{p}$ regularity, computing the so-called $p$-exponent. We prove that in the general case the Hölder and $p$ exponent are different at each point. We also compute the dimension of the sets where the functions have a given pointwise regularity and prove that these functions are multifractal both from the point of view of Hölder and $L^{p}$ local regularity with different spectra of singularities. Furthermore, we check that multifractal formalism type formulas hold for functions in that family.
\end{abstract}

Keywords: Multifractal analysis, pointwise regularity, wavelet bases, fractionnal derivatives.

Mathematics Subject Classification: 26A16, 26A33, 26B35, 42C40.

\footnotetext{
*Email: claire.coiffard@gmail.com

$\dagger$ Email: clothilde.melot@univ-amu.fr

‡Email: thomas.willer@univ-amu.fr
} 


\section{Introduction}

Multifractal analysis for signal analysis was elaborated in the context of fully developped turbulence in order to study the signal of velocity of turbulent fluid, whose regularity is changing from point to point.

Indeed one criterium for estimating the pointwise regularity of a signal at a point $x_{0}$ is to compute the pointwise Hölder exponent. Recall its definition.

Definition 1. Let $x_{0} \in \mathbb{R}^{d}$ and $\alpha \geq 0$.

$A$ locally bounded function $f: \mathbb{R}^{d} \rightarrow \mathbb{R}$ belongs to $C^{\alpha}\left(x_{0}\right)$ if there exists $C>0$ and a polynomial $P_{x_{0}}$ with $\operatorname{deg}(P) \leq[\alpha]$ and such that on a neighborhood of $x_{0}$,

$$
\left|f(x)-P_{x_{0}}(x)\right| \leq C\left|x-x_{0}\right|^{\alpha} .
$$

The pointwise Hölder exponent of $f$ at $x_{0}$ is $h_{f}\left(x_{0}\right)=\sup \left\{\alpha: f \in C^{\alpha}\left(x_{0}\right)\right\}$.

Under these assumptions, let $\alpha \in \mathbb{R}$ be fixed and set $E_{f}(\alpha)=\left\{x_{0}: h_{f}\left(x_{0}\right)=\alpha\right\}$.

Performing the multifractal analysis of the signal $f$ is computing for every $\alpha$ the Hausdorff dimension $d_{f}(\alpha)$ of the set $E_{f}(\alpha)$.

If $E_{f}(\alpha)$ is non empty for more than two values of $\alpha$ the function is called a multifractal function (if it is not empty for one single value of $\alpha$ it will be called a monofractal function). The map $\alpha \mapsto d_{f}(\alpha)$ is called the spectrum of Hölder singularities of the function $f$.

For example one can see that the classical Weierstrass function

$$
f: x \mapsto \sum_{n \in \mathbb{N}} A^{n} \cos \left(B^{n} x\right) \quad \text { with } A B>1 \text { and } A<1
$$


is a monofractal function, indeed $d_{f}(\alpha)=-\infty$ for $\alpha \neq-\frac{\ln (A)}{\ln (B)}$ and 1 for $\alpha=-\frac{\ln (A)}{\ln (B)}$. Other examples can be found in numerous works and studies of multifractal functions (see [17] for references).

Other types of pointwise singularities can be studied. Calderon and Zygmund in [4] introduced a local exponent based on $L^{p}$ norms, the " $p$ exponent".

Definition 2. Let $p \in[1, \infty]$ and $u$ such that $u \geq-\frac{d}{p}$. Let $f$ be a function in $L_{\text {loc }}^{p}$. The function $f$ belongs to $T_{u}^{p}\left(x_{0}\right)$ if there exists $R>0, P$ a polynomial such that $\operatorname{deg}(P) \leq u$, and $C>0$ such that

$$
\forall \rho \leq R:\left(\frac{1}{\rho^{d}} \int_{\left|x-x_{0}\right| \leq \rho}|f(x)-P(x)|^{p} d x\right)^{\frac{1}{p}} \leq C \rho^{u} .
$$

The p-exponent of $f$ at $x_{0}$ is $u_{f}^{p}\left(x_{0}\right)=\sup \left\{u: f \in T_{u}^{p}\left(x_{0}\right)\right\}$

Under these assumptions, let $\alpha \in \mathbb{R}$ be fixed. We denote $E_{f, p}(\alpha)=\left\{x_{0}\right.$ : $\left.u_{f}^{p}\left(x_{0}\right)=\alpha\right\}$ and $d_{f, p}(\alpha)$ the Hausdorff dimension of $E_{f, p}(\alpha)$. The map $\alpha \mapsto$ $d_{f, p}(\alpha)$ is called the spectrum of p-singularities of the function $f$.

The $p$ exponent was studied in the context of multifractal analysis in [13], [12] for instance, but as far as we know there were not many contributions where examples of multifractal functions were studied from this point of view. The work of [8] proves that generically (in the sense of prevalence) in a given functional space like a Sobolev or Besov space, the spectrum of Hölder singularities and the one of $p$ singularities coincide. Indeed the author proves the existence of a prevalent set of functions in a given Sobolev or Besov space (i.e a set whose complement is a Haar-null set) which have the same spectra. It is actually possible to exhibit families of functions which do not belong to this prevalent set. 
This is the case of the functions we will study.

Let us see that one can easily build functions were the two pointwise exponents are different at least at one given point. For example let $\alpha>0$ and the function $x \mapsto g(x)=|x|^{\alpha} \sum_{j=1}^{\infty} I_{D_{j}}(x)$ with $D_{j}=\left[1 / 2^{j}-1 / 2^{3 j}, 1 / 2^{j}\right]$ for $j \geq 0$. The function $g$ satisfies $h_{g}(0)=\alpha<u_{g}^{p}(0)=\alpha+1 / p$ for any $p \geq 1$.

In this work we study a slight modification of the family of functions introduced by S. Jaffard [16], and prove that for each member of this family the spectrum of Hölder singularities and the one of $p$ singularities are different. More precisely we will compute at each point of $\mathbb{R}$ the pointwise Hölder exponent, as well as the $p$-exponent. Whenever the two exponents are different, this is the signature of an oscillating behavior of "chirp" type (see details in Section 4.2 and also [10], [11] on oscillating behaviors and wavelets) and we will prove that oscillating exponents that detect this kind of behavior are non trivial.

Furthermore we will also check that the spectra of singularities satisfy multifractal formalism type formulas. These formulas are generally heuristic formulas whose goal is to compute the spectrum of singularities with the help of global quantities. The first one was introduced by Frish and Parisi in the context of fully developped turbulence [19]. It was then rewritten using wavelet analysis by A. Arnéodo and al. [2]. The domain of validity, counter examples and generic properties of this kind of formulas were the subjects of studies and they are still active fields [20]. We will focus on the multifractal type formulas described in [14] since they fit with the kind of singularities we study. We will prove that these formulas are satisfied by our signals. 
The content of the paper is as follows. We present some preliminary notions on wavelets and Hausdorff dimension in Section 2, then the family of functions under study in Section 3, and the main results of the paper in Section 4. Afterwards we provide developments and proofs of the main results separately: local regularity, spectra of singularities, and multifractal formalism are adressed in Section 5, 6 and 7 respectively.

\section{Definitions and notations}

\section{$2.1 \quad$ Wavelet basis}

In all the following $\Lambda$ denotes the set of all dyadic intervals $\lambda=\left[k 2^{-j},(k+1) 2^{-j}[\right.$, $j \in \mathbb{Z}, k \in \mathbb{Z}$ and $\Lambda_{j}$ with $j \in \mathbb{Z}$ the subset of dyadic intervals $\lambda$ of the type $\lambda=\left[k 2^{-j},(k+1) 2^{-j}[\right.$ with $k \in \mathbb{Z}$. We will sometimes write $\lambda=(j, k)$ if no confusion is possible. The notation $[x]$ significates that $[x]$ is the integer part of $x$, and $\lceil x\rceil$ denotes the smallest integer not less than $x$.

Recall that a wavelet basis is a set of functions such that $\phi$ and $\psi$ are functions in $L^{2}(\mathbb{R})$ and satisfy $\Psi=\{\phi(.-k), k \in \mathbb{Z}\} \bigcup\left\{2^{j / 2} \psi\left(2^{j} .-k\right), j \geq 0 ; k \in \mathbb{Z}\right\}$ is an orthonormal basis of $L^{2}(\mathbb{R})$. We consider a wavelet basis regular enough, i.e $\phi$ and $\psi$ are functions in $C^{r+1}(\mathbb{R})$ with compact support. We will call $r$ the regularity of the basis $\Psi$. This is always possible (see for example [5] for such constructions).

In order to simplify the notations we will write $\psi_{\lambda}(x)=2^{j / 2} \psi\left(2^{j} x-k\right)$.

To sum up we have the following equality in $L^{2}(\mathbb{R})$ 


$$
\forall f \in L^{2}(\mathbb{R}), f(x)=\sum_{k \in \mathbb{Z}} c_{k} \phi(x-k)+\sum_{j \geq 0} \sum_{\lambda \in \Lambda_{j}} c_{\lambda} \psi_{\lambda}(x)
$$

with

$$
\begin{aligned}
c_{k} & =\int f(x) \phi(x-k) d x, \forall k \in \mathbb{Z} \\
c_{j, k}=c_{\lambda} & =\int f(x) \psi_{\lambda}(x) d x .
\end{aligned}
$$

\subsection{Hausdorff dimension}

Definition 3. Let $A \subset \mathbb{R}^{d}$; if $\varepsilon>0$, an $\varepsilon$-covering of $A$ is a countable collection $R=\left\{A_{i}\right\}_{i \in \mathbb{N}}$ such that each diameter $\left|A_{i}\right|$ is less than $\varepsilon$, and $A \subset \bigcup_{i=1}^{\infty} A_{i}$. If $\delta \in[0, d]$, set

$$
M_{\varepsilon}^{\delta}=\inf _{R}\left(\sum_{i}\left|A_{i}\right|^{\delta}\right),
$$

where the infimum is taken on all $\epsilon$-coverings $R$.

For any $\delta \in[0, d]$, the $\delta$-dimensional Hausdorff measure of $A$ is $\operatorname{mes}_{\delta}(A)=$ $\lim _{\varepsilon \rightarrow 0} M_{\varepsilon}^{\delta}$. There exists $\delta_{0} \in[0, d]$ such that

$$
\forall \delta<\delta_{0}, \quad \operatorname{mes}_{\delta}(A)=+\infty \quad \text { and } \forall \delta>\delta_{0}, \quad \operatorname{mes}_{\delta}(A)=0 ;
$$

this critical $\delta_{0}$ is the Hausdorff dimension of $A$, and will be denoted by $\operatorname{dim}(A)$.

Suppose that $A$ is a subset of $\mathbb{R}^{d}$, and that a numerical quantity $\mathcal{H}(x)$ taking values in $\mathbb{R}^{m}$ is attached to each point of $A$. If $\mathcal{H}(x)$ has no regularity, then the level sets of $\mathcal{H}$

$$
E_{H}=\{x: \mathcal{H}(x)=H\}
$$

may be fractal sets. We consider here the special case where $\mathcal{H}(x)$ is the pointwise Hölder exponent at point $x$ of a function $f$, i.e $h_{f}(x)$, and for $p \geq 1$ the case where $\mathcal{H}(x)$ is the $p$-exponent at point $x$ of a function $f$, i.e $u_{p}^{f}(x)$. Thus 
we focus on two spectra, which are defined as follows.

Definition 4. For some function $f$, we define the Hölder spectrum by:

$$
d_{f}: h \mapsto \operatorname{dim}\left(E_{h}\right), \text { with } E_{h}=\left\{x: h_{f}(x)=h\right\},
$$

and the $p$ spectrum by:

$$
d_{f}^{p}: u \mapsto \operatorname{dim}\left(E_{u}^{p}\right), \text { with } E_{u}^{p}=\left\{x: u_{p}^{f}(x)=u\right\}
$$

\section{Functions under study}

We define a family of functions $f$ on $[0,1]$ as a modification of a model by Jaffard [16]. The functions in this family depend on three parameters $\alpha, \beta$ and $\gamma$, with $\alpha \geq 1$ and $\beta \geq 1$ integers and $\gamma>0$ a non integer. We set

$$
f(x)=\sum_{\lambda \in \Lambda(\alpha, \beta)} 2^{-(\gamma+1 / 2) j} \psi_{\lambda}(x)
$$

In (5), $\Lambda(\alpha, \beta)=\bigcup_{m \geq 1} \Lambda_{m}^{(\alpha, \beta)}$, where $\Lambda_{m}^{(\alpha, \beta)}$ is the set of $\lambda=(j, k)$ such that

- $j=\alpha \beta m, m>1$,

- $2^{-j} k=\varepsilon_{1} \ell_{1}+\ldots+\varepsilon_{m-1} \ell_{m-1}+\varepsilon_{m}^{\prime} \ell_{m}^{\prime}$, where $\varepsilon_{1}=1$, and for each $i>1$ $\varepsilon_{i}= \pm 1, \varepsilon_{i}^{\prime}= \pm 1, \ell_{i}=2^{-i}$ and $\ell_{i}^{\prime}=2^{-\alpha i}$.

In the sequel, we will denote by $c_{\lambda}$ the wavelet coefficients of the function $f$ :

$$
c_{\lambda}=<f, \psi_{\lambda}>
$$


Let us notice that for $\alpha=1$, the function is the same as the one of Jaffard [16].

Remark that the definition of $f$ implies that each $m>1$ creates $2^{m-1}$ non vanishing coefficients identified by the dyadics $\frac{k}{2^{m-1}} \pm \frac{1}{2^{\alpha m}}, k$ odd, and their scale $\alpha \beta m$. Its values are all equal to $2^{-(\gamma+1 / 2) \alpha \beta m}$.

- In the special case where $\alpha=\beta=1$, this means that at each scale $j$ all the irreducible fractions $\frac{k}{2^{j}}, k$ odd, yield non vanishing wavelet coefficients.

- In the case where $\beta=1$ and $\alpha=2$ each fraction $\frac{k}{2^{m-1}} \pm \frac{1}{2^{\alpha m}}, k$ odd, is an irreducible fraction at scale $j=\alpha m$.

This amounts to say that the non vanishing coefficients appear at scale $j=\alpha m$ on irreducible fractions which can be written $\frac{k}{2^{j}}=\frac{K}{2^{m-1}} \pm \frac{1}{2^{\alpha m}}$.

Figure 1 provides the repartition of the wavelet coefficients at scale $j=$ $1, . ., 6$ with $\alpha=2$ and $\beta=1$.

- In case $\beta>1$ the fraction of type $\frac{K}{2^{m-1}} \pm \frac{1}{2^{\alpha m}}$ is no more irreducible at scale $j=\alpha \beta m$. The coefficient will appear at a finer scale than scale $\alpha m$. Figure 2 gives an insight of this situation for $\alpha=\beta=2$.

Following the characterization of $C^{\gamma}(\mathbb{R})$ (see for example [17]) with the help of wavelet coefficients we have $f \in C^{\gamma}(\mathbb{R})$ since its wavelet coefficients satisfy: for all $j \geq 0$ and all dyadic interval $\lambda \in \Lambda_{j},\left|c_{\lambda}\right| \leq 2^{-j(\gamma+1 / 2)}$.

Remark also that $f$ is compactly supported, thus it is in all $L^{p}$ spaces for $p \geq 1$. Following [9] (see Chapter 5 Section 5.3), and the fact that $f$ is bounded and in $C^{\gamma}(\mathbb{R})$, we get that the serie in $(5)$ converges in all $L^{p}$ for $1 \leq p \leq \infty$. 


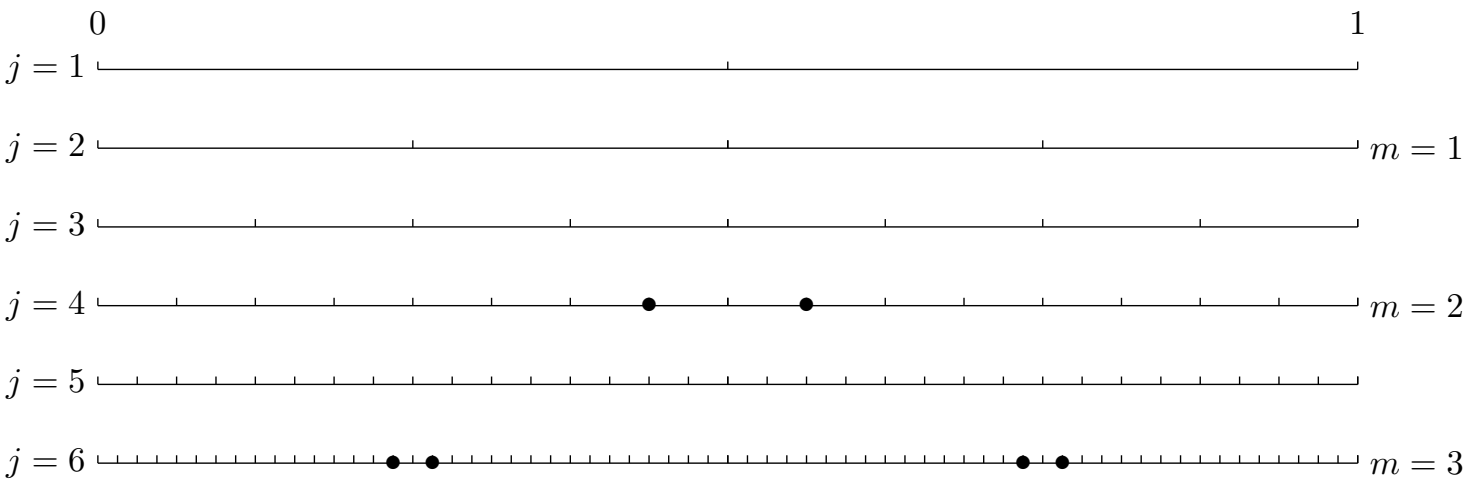

Figure 1: Case $\beta=1$ and $\alpha=2$. The non vanishing wavelet coefficients appear on dyadic points $\frac{k}{2^{j}}=\frac{K}{2^{m-1}} \pm \frac{1}{2^{\alpha m}}$ with $K$ odd and $j=\alpha m$.

- if we choose $m=2$ and $j=4$, then $\frac{k}{2^{j}}=\varepsilon_{1} \ell_{1}+\varepsilon_{2}^{\prime} \ell_{2}^{\prime}=\frac{1}{2} \pm \frac{1}{2^{4}}$ yields non vanishing coefficients at scale $j=4$.

- if we choose $m=3$ and $j=6$, then $\frac{k}{2^{j}}=\varepsilon_{1} \ell_{1}+\varepsilon_{2} \ell_{2}+\varepsilon_{3}^{\prime} \ell_{3}^{\prime}=\frac{1}{2} \pm \frac{1}{4} \pm \frac{1}{2^{6}}$ yields non vanishing coefficients at scale $j=6$.

0

$j=1$

$j=2$

$j=3$

$j=4$

$j=5$

$j=6$

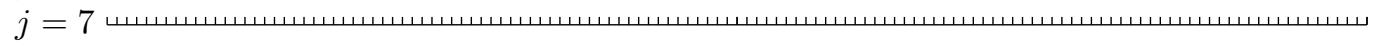

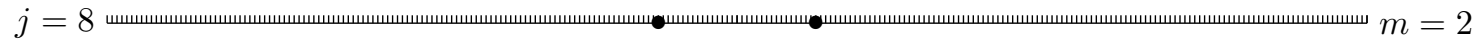

$j=9$ h

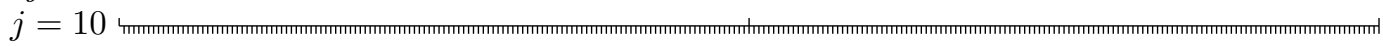

$j=11$ h

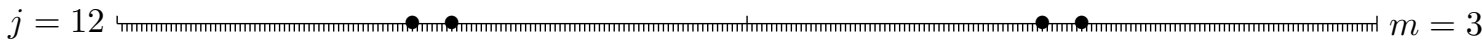

Figure 2: Case $\beta=2$ and $\alpha=2$. The non vanishing wavelet coefficients appear on dyadic points $\frac{k}{2^{j}}=\frac{K}{2^{m-1}} \pm \frac{1}{2^{\alpha m}}$ with $K$ odd and $j=\alpha \beta m$. 


\section{Results}

\subsection{Hölder and $p$-singularities}

One of the key point in multifractal analysis of a function is to understand the underlying structure of the sets $E_{h}$ and $E_{u}^{p}$. It turns out as we will see in the following that there is a deep connection between these sets and sets of points approximated by special sequences of dyadics. Let us introduce what we mean by these "special sequences".

Let $\alpha$ be an integer larger or equal than 1 , and $\mathcal{S}_{\alpha}$ the set of dyadic points such that $\frac{k^{\prime}}{2^{j^{\prime}}} \in \mathcal{S}_{\alpha}$ if one can find $(j, k)$ such that $\frac{k^{\prime}}{2^{j^{\prime}}}=\frac{k}{2^{j-1}} \pm \frac{1}{2^{\alpha j}}$ with $\frac{k}{2^{j-1}}$ an irreducible fraction of order $j-1$. The set $\mathcal{S}_{\alpha}$ describes exactly the locations of non vanishing wavelet coefficients in $f$.

We will need the rate of approximation of $x_{0}$ by dyadics in this set $\mathcal{S}_{\alpha}$ given by

$$
r_{\alpha}\left(x_{0}\right)=\limsup _{j^{\prime} \rightarrow \infty} \frac{\log \left(\left|K_{j^{\prime}}\left(x_{0}\right) 2^{-j^{\prime}}-x_{0}\right|\right)}{\log \left(2^{-j}\right)}
$$

where $K_{j^{\prime}}\left(x_{0}\right)=\operatorname{argmin}_{k / k 2^{-j^{\prime}} \in \mathcal{S}_{\alpha}}\left(\left|x_{0}-k 2^{-j^{\prime}}\right|\right)$ and $\left(j, k_{j-1}\left(x_{0}\right)\right)$ the integers such that $\frac{K_{j^{\prime}}\left(x_{0}\right)}{2^{j^{\prime}}}=\frac{k_{j-1}\left(x_{0}\right)}{2^{j-1}} \pm \frac{1}{2^{\alpha j}}$ with $\frac{k_{j-1}\left(x_{0}\right)}{2^{j-1}}$ an irreducible fraction.

Since we always have

$$
\left|K_{j^{\prime}}\left(x_{0}\right) 2^{-j^{\prime}}-x_{0}\right| \leq 2^{-(j-1)}
$$

then $r_{\alpha}\left(x_{0}\right) \geq 1$.

Remark that dyadic points satisfy exactly $r_{\alpha}\left(x_{0}\right)=1$. Indeed if $x_{0}=\frac{K_{0}}{2^{j_{0}}}$ is a 
dyadic point then for $j^{\prime}$ large enough $\left|x_{0}-K_{j^{\prime}}\left(x_{0}\right) 2^{-j^{\prime}}\right|=\frac{1}{2^{j-1}}-\frac{1}{2^{\alpha j}}$, which yields $r_{\alpha}\left(x_{0}\right)=1$.

Given the definition of $r_{\alpha}\left(x_{0}\right)$, for every $\delta>0$ there exists a subsequence $m_{n}^{\prime}$ $\left(m_{n}^{\prime} \rightarrow \infty\right.$ when $\left.n \rightarrow \infty\right)$ and $m_{n}$, with $\frac{K_{m_{n}^{\prime}}\left(x_{0}\right)}{2^{m_{n}^{\prime}}}=\frac{k_{m_{n}-1}}{2^{m_{n}-1}} \pm \frac{1}{2^{\alpha m_{n}}}$ such that,

$$
\left|K_{m_{n}^{\prime}}\left(x_{0}\right) 2^{-m_{n}^{\prime}}-x_{0}\right|<2^{-m_{n}\left(r_{\alpha}\left(x_{0}\right)-\delta\right)}
$$

Furthermore, still using the definition of $r_{\alpha}\left(x_{0}\right)$, for every $\varepsilon>0$, there exists a constant $M>0$ such that for all $m^{\prime} \geq M$, there is $m \geq M / \alpha$

$$
\left|K_{m^{\prime}}\left(x_{0}\right) 2^{-m^{\prime}}-x_{0}\right|>2^{-m\left(r_{\alpha}\left(x_{0}\right)+\varepsilon\right)} .
$$

We can now give the results we want to prove in the following.

Theorem 1. Let $\alpha, \beta$ and $\gamma$, with $\alpha \geq 1$ and $\beta \geq 1$ two integers and $\gamma>0$ a non integer. Let $p \geq 1$.

- Suppose $x_{0} \in[0,1]$ and $r_{\alpha}\left(x_{0}\right) \leq \alpha \beta$ then $h_{f}\left(x_{0}\right)=\frac{\alpha \beta \gamma}{r_{\alpha}\left(x_{0}\right)}$ and $u_{f}^{p}\left(x_{0}\right)=\frac{\alpha \beta \gamma}{r_{\alpha}\left(x_{0}\right)}+\left(\frac{\alpha \beta}{r_{\alpha}\left(x_{0}\right)}-1\right) \frac{1}{p}$.

- Suppose $x_{0} \in[0,1]$ and $r_{\alpha}\left(x_{0}\right)>\alpha \beta$ then $h_{f}\left(x_{0}\right)=\alpha \beta \gamma$ and $u_{f}^{p}\left(x_{0}\right)=$ $\alpha \beta \gamma+\frac{\alpha \beta-1}{p}$.

- $x_{0} \notin[0,1]$ then $h_{f}\left(x_{0}\right)=u_{f}^{p}\left(x_{0}\right)=+\infty$.

As a corollary we get

Corollary 2. The Hölder spectrum of $f$ is the function $d_{f}$ defined on the interval $[\gamma, \alpha \beta \gamma]$ such that $d_{f}(h)=\frac{h}{\alpha \beta \gamma}$. The $p$ spectrum is the function $d_{f, p}$ defined on the interval $\left[\gamma, \alpha \beta \gamma+\frac{\alpha \beta-1}{p}\right]$ and such that $d_{f, p}(u)=\frac{u+\frac{1}{p}}{\alpha \beta\left(\gamma+\frac{1}{p}\right)}$. 
The proof of Theorem 1 is given in Section 5 and the one of Corollary 2 in Section 6 .

The results on the multifractal formalisms can be found in Section 7 .

\subsection{Oscillation singularities}

Our main theorem proves that whenever $x_{0}$ is such that $r_{\alpha}\left(x_{0}\right) \neq \alpha \beta$ we have $h_{f}\left(x_{0}\right)<u_{f}^{p}\left(x_{0}\right)$. We will prove that the singularity at $x_{0}$ is an oscillating one by computing the oscillating singularity exponent. This will precise quantitatively the behavior of the function at $x_{0}$.

Let us first explain what we mean by "oscillating exponent". We first give some notations.

Let $t>0$ and let $h_{f}^{t}\left(x_{0}\right)$ denote the Hölder exponent of the fractional primitive of order $t$ at $x_{0}$ of a function $f \in L_{l o c}^{\infty}$. More precisely let $\phi$ be a $C^{\infty}$ compactly supported function satisfying $\phi\left(x_{0}\right)=1$. Let $(I d-\Delta)^{-t / 2}$ be the convolution operator which amounts to multiply the Fourier transform of the function with $\left(1+|\xi|^{2}\right)^{-t / 2}$; we denote by $h_{f}^{t}\left(x_{0}\right)$ the Hölder exponent at $x_{0}$ of the function $f^{t}=(I d-\Delta)^{-t / 2}(\phi f)$. The following definition was introduced in [1] (see also [15], where alternative definitions are discussed).

Definition 5. Let $f: \mathbb{R}^{d} \rightarrow \mathbb{R}$ be a bounded function. If $h_{f}\left(x_{0}\right) \neq+\infty$, then the oscillation exponent of $f$ at $x_{0}$ is defined by

$$
\beta_{f}\left(x_{0}\right)=\left(\frac{\partial}{\partial t} h_{f}^{t}\left(x_{0}\right)\right)_{t=0}-1
$$

(where the derivative at $t=0$ should be understood as a right-derivative). 
Note that the mapping $t \longrightarrow h_{f}^{t}\left(x_{0}\right)$ is a concave increasing function [1]. So that the derivative in (10) always exists (but may be infinite).

One can check that for the function $f: x \mapsto|x|^{\alpha} \sin \left(\frac{1}{|x|^{\beta+1}}\right)$ with $\alpha>0$ and $\beta>0$ we have $h_{f}\left(x_{0}\right)=\alpha$ and $\beta_{f}\left(x_{0}\right)=\beta>0$ (see [15] for more details on chirp-type behavior in a wavelet basis-setting). This example is typical for the kind of behavior we wish to describe with the oscillating singularity exponent.

We will see that we can derive directly the computation of the oscillating singularity exponent from the previous theorem.

Proposition 1. Let $\alpha, \beta$ and $\gamma$, with $\alpha \geq 1$ and $\beta \geq 1$ two integers and $\gamma>0$ a non integer.

- Suppose $x_{0} \in[0,1]$ and $r_{\alpha}\left(x_{0}\right) \leq \alpha \beta$ then $\beta_{f}\left(x_{0}\right)=\frac{\alpha \beta}{r_{\alpha}\left(x_{0}\right)}-1$.

- Suppose $x_{0} \in[0,1]$ and $r_{\alpha}\left(x_{0}\right)>\alpha \beta$ then $\beta_{f}\left(x_{0}\right)=\alpha \beta-1$.

- $x_{0} \notin[0,1]$ then $\beta_{f}\left(x_{0}\right)=0$.

If $x_{0} \in[0,1]$ the critical case $r_{\alpha}\left(x_{0}\right)=\alpha \beta$ is the only one for which $\beta_{f}\left(x_{0}\right)=0$.

As a corollary we can compute the dimension of the set of points $E_{\left(h, \beta_{o}\right)}=\left\{x_{0}\right.$ : $\left.\left(h_{f}\left(x_{0}\right), \beta_{f}\left(x_{0}\right)\right)=\left(h, \beta_{o}\right)\right\}$ that we will denote $d\left(h, \beta_{o}\right)$.

Corollary 3. Let $\alpha, \beta$ and $\gamma$, with $\alpha \geq 1$ and $\beta \geq 1$ two integers and $\gamma>0$ a non integer.

The function $\left(h, \beta_{o}\right) \mapsto d\left(h, \beta_{o}\right)$ is supported on the segment $h=\left(\beta_{o}+1\right) \gamma$ with $\beta_{o} \in[0, \alpha \beta-1]$ and on this segment $d\left(h, \beta_{o}\right)=\frac{h}{\alpha \beta \gamma}=\frac{\beta_{o}+1}{\alpha \beta}$. 


\section{$5 \quad$ Local regularity and wavelet coefficients}

\subsection{Hölder regularity}

We first want to study the pointwise Hölder regularity of the function $f$ at each point $x_{0} \in \mathbb{R}$. This amounts to compute the pointwise Hölder exponent at $x_{0} \in \mathbb{R}$. Recall that we can apply Theorem 1 of [17] which relates the so called wavelet leaders, which depend on the wavelet coefficients of $f$, with the pointwise Hölder exponent at $x_{0}$. We need to start with a definition.

Definition 6. [17] Two dyadic intervals $\lambda_{1}$ and $\lambda_{2}$ are called adjacent if they are at the same scale and if dist $\left(\lambda_{1}, \lambda_{2}\right)=0$ (note that a dyadic interval is adja-

cent to himself). We denote by $\lambda_{j}\left(x_{0}\right)$ the dyadic interval of size $2^{-j}$ containing $x_{0}$ and $3 \lambda_{j}\left(x_{0}\right)$ the set of 3 dyadic intervals adjacent to $\lambda_{j}\left(x_{0}\right)$.

More precisely if $\lambda=(j, k)$ then we denote $\lambda^{l}=(j, k-1)$ and $\lambda^{r}=(j, k+1)$.

Then let

$$
d_{j}\left(x_{0}\right)=\sup _{\lambda \in 3 \lambda_{j}\left(x_{0}\right)} d_{\lambda}
$$

with

$$
d_{\lambda}=\sup _{\lambda^{\prime} \subset \lambda}\left|2^{j^{\prime} / 2} c_{\lambda^{\prime}}\right|
$$

$d_{\lambda}$ is called a "wavelet leader".

Theorem 4. [17] Let $\delta>0, x_{0} \in \mathbb{R}$ and $f$ be a function in $L^{\infty}(\mathbb{R})$. Suppose $\Psi$ is a wavelet basis of regularity $r>[\delta]+1$.

- Suppose $f$ is in $C^{\delta}\left(x_{0}\right)$. Then there exists $C>0$ such that

$$
\forall j \geq 0, d_{j}\left(x_{0}\right) \leq C 2^{-\delta j}
$$


- Conversely suppose that (13) holds and furthermore there exists $\varepsilon>0$ such that $f \in C^{\varepsilon}(\mathbb{R})$. Then $f$ belongs to $C^{\delta^{\prime}}\left(x_{0}\right)$ for all $\delta^{\prime}<\delta$. In particular this means that $h_{f}\left(x_{0}\right) \geq \delta$.

- Suppose $f \in C^{\varepsilon}(\mathbb{R})$. Then $h_{f}\left(x_{0}\right)=\liminf _{j \rightarrow \infty} \frac{\ln \left(d_{j}\left(x_{0}\right)\right)}{\ln \left(2^{-j}\right)}$.

Since $f$ belongs to $C^{\gamma}(\mathbb{R})$ we only need to compute, at each point $x_{0}, d_{j}\left(x_{0}\right)$ at each scale $j \geq 0$. This is what is done in Section 5.4.

\section{2 $\quad L^{p}$ pointwise regularity}

To study and compute the $p$ exponent at each point $x_{0}$ in $\mathbb{R}$, we also compute some quantities related to wavelet coefficients.

Define the so-called $p$ leader

$$
D_{\lambda, p}=\left(\sum_{\lambda^{\prime} \subset \lambda}\left|c_{\lambda^{\prime}}\right|^{p} 2^{j^{\prime}\left(\frac{p}{2}-1\right)}\right)^{1 / p}
$$

We set

$$
D_{j, p}\left(x_{0}\right)=\left(\sum_{\lambda^{\prime} \subset 3 \lambda_{j}\left(x_{0}\right)}\left|c_{\lambda^{\prime}}\right|^{p} 2^{j^{\prime}\left(\frac{p}{2}-1\right)}\right)^{1 / p},
$$

with the notation $\lambda^{\prime}=\left(j^{\prime}, k^{\prime}\right)$.

It is easy to see that actually $D_{j, p}\left(x_{0}\right)=\left(\sum_{\lambda \in 3 \lambda_{j}\left(x_{0}\right)} D_{\lambda, p}^{p}\right)^{1 / p}$.

Before stating the characterization theorem of [14], we need to recall the characterization of Besov spaces $B_{p}^{s, p}[18]$.

Theorem 5. Let $s \in \mathbb{R}$ and $\infty>p>0, q>0$ and $r$ an integer such that $r>[s]+1$. Let $\Psi$ be a regular wavelet basis.

Suppose $f$ is a tempered distribution with $c_{k}, k \in \mathbb{Z},\left(c_{j k}\right)_{j \geq 0 ; k \in \mathbb{Z}}$ its wavelet coefficients defined by (4). 
A tempered distribution $f$ belongs to $B_{p}^{s, p}$ if $\left(c_{k}\right)$ belongs to $l^{p}$ and if

$$
\sum_{j \geq 0} \sum_{k}\left|c_{j, k} 2^{(s+1 / 2-1 / p) j}\right|^{p}<+\infty
$$

Remark that a compactly supported function in $C^{\varepsilon}(\mathbb{R})$ belongs to any Besov space $B_{p}^{s, p}$ for $s<\varepsilon$.

We have the following theorem of [14] in a slightly modified version in comparison to the original one

Theorem 6. Let $p \geq 1$ and $u>\frac{-1}{p}$. Let $\Psi$ a r regular wavelet basis with $r \geq[u]+1$.

- Suppose $f$ belongs to $T_{u}^{p}\left(x_{0}\right)$ then there exists a constant $C>0$ such that for all $j \geq 0$

$$
D_{j, p}\left(x_{0}\right) \leq C 2^{-j(u+1 / p)}
$$

- Suppose $f$ belongs to $B_{p}^{\delta, p}$ for some $\delta>0$. If there exists a constant $C>0$ such that (17) holds for all $j \geq 0$ then $f \in T_{u^{\prime}}^{p}\left(x_{0}\right)$ for all $u^{\prime}<u$.

- Suppose $f \in B_{p}^{\delta, p}$ for some $\delta>0$. Then $u_{p}^{f}\left(x_{0}\right)=\liminf _{j \rightarrow \infty} \frac{\ln \left(D_{j, p}\left(x_{0}\right)\right)}{\ln \left(2^{-j}\right)}-\frac{1}{p}$.

Remark that whenever the Hölder exponent at a point $x_{0}$ of a function $f$ is defined, and if this function satisfies the hypothesis of Definitions 1 and 2 we immediately have

$$
h_{f}\left(x_{0}\right) \leq u_{f}^{p}\left(x_{0}\right)
$$




\subsection{Oscillating singularities}

Exactly as previously we can define the $s$-wavelet leader for $s>0$

$$
d_{j}^{s}\left(x_{0}\right)=\sup _{\lambda^{\prime} \subset 3 \lambda_{j}\left(x_{0}\right)}\left|2^{j^{\prime}\left(-s+\frac{1}{2}\right)} c_{\lambda^{\prime}}\right|, \quad(s-\text { leader }) .
$$

The following characterization holds, derived from Theorem 4 .

Proposition 2. Let $f$ be in $C^{\varepsilon}(\mathbb{R})$ for $\epsilon>0$. Then $h_{f}^{s}\left(x_{0}\right)=\liminf _{j \rightarrow \infty} \frac{\ln \left(d_{j}^{s}\left(x_{0}\right)\right)}{\ln \left(2^{-j}\right)}$.

\subsection{Study of the pointwise regularity of the function $f$}

We give in the next section 5.4.1 the proof of Theorem 1 in the case $\beta=1$. For seek of completeness, the modifications needed to be done when $\beta>1$ are given in section 5.4.2. We end up by proving in Section 5.4.3 Proposition 1 as a consequence of Theorem 1 .

In all what follows we have $p \geq 1$ and denote $a p=\gamma p+1$.

\subsubsection{Case $\beta=1$}

Wavelet and $p$ leaders Let $\lambda$ be a dyadic interval indexed by $(j, k)$. Let $m_{1}$ be an integer such that $\alpha\left(m_{1}-1\right)<j \leq \alpha m_{1}$.

1 Suppose $\frac{k}{2^{j}}=\frac{K}{2^{m^{\prime}}}$ is an irreducible fraction with $m^{\prime} \geq m_{1}$. It means that the coefficient associated with the irreducible fraction will appear at scale $\alpha\left(m^{\prime}+1\right) \geq$ j. So this coefficient will be the first non vanishing coefficient. Then $d_{\lambda}=$ $\sup _{\lambda^{\prime} \subset \lambda}\left|2^{j^{\prime} / 2} c_{\lambda}^{\prime}\right|=2^{-\alpha \gamma\left(m^{\prime}+1\right)}$.

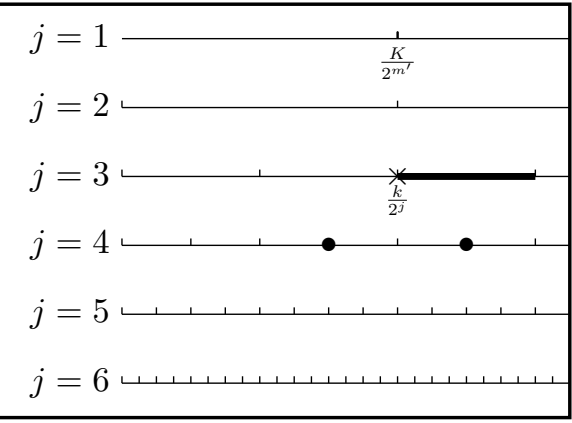


For what concerns the $p$-leaders, we already talked about the first nonvanishing coefficient. The following coefficient is located in $\frac{k}{2^{j}}+\frac{1}{2^{j}}$ and appears at scale $\alpha(j+1)$ if $m<j$, and if $m=j$ at scale $\alpha(j+2)$ at location $\frac{k}{2^{j}}+\frac{1}{2^{j+1}}-\frac{1}{2^{\alpha(j+2)}}$. We get in all cases

$$
2^{-a p \alpha\left(m^{\prime}+1\right)} \leq D_{\lambda, p}^{p} \leq 2^{-a p \alpha\left(m^{\prime}+1\right)}+\sum_{\ell=j+1}^{\infty} 2^{\ell-(j+1)} 2^{-a p \alpha \ell}=2^{-a p \alpha\left(m^{\prime}+1\right)}+C 2^{-a p \alpha j}
$$

2 Now suppose that $\frac{k}{2^{j}}=\frac{K}{2^{m^{\prime}}}$ is an irreducible fraction with $m^{\prime}<m_{1}$. That means that the coefficient associated with this irreducible fraction appears at scale $\alpha m^{\prime}<j$, so before the scale we consider. But in that case, we can notice that $\frac{k}{2^{j}}+\frac{1}{2^{j}}$ is irreducible and one of the corresponding coefficient appears at location $\frac{k}{2^{j}}+\frac{1}{2^{j}}-\frac{1}{2^{\alpha(j+1)}}$. This yields

$$
\begin{aligned}
& j=1 \\
& j=2 \\
& j=3 \\
& j=4 \\
& j=5 \\
& j=6
\end{aligned}
$$
$d_{\lambda}=2^{-\alpha \gamma(j+1)}$.

Again, the $p$-leader is simply given by

$$
D_{\lambda, p}^{p}=2^{-a p \alpha(j+1)}+\sum_{\ell=j+2}^{\infty} 2^{\ell-(j+2)} 2^{-a p \alpha \ell}=C 2^{-a p \alpha j}
$$

Computation of the local regularity of $f \quad$ Let us now prove Theorem 1 in case $\beta=1$. Let $x_{0} \in \mathbb{R}$ and $p \geq 1$.

1. Let us first remark that for $x_{0} \notin[0,1]$, we have for $j$ large enough $d_{j}\left(x_{0}\right)=0=D_{j, p}\left(x_{0}\right)$ since for $j$ large enough all the wavelet coefficients adjacent to $\lambda_{j}\left(x_{0}\right)$ are vanishing. Thus $h_{f}\left(x_{0}\right)=u_{p}^{f}\left(x_{0}\right)=+\infty$. 
2. Let $x_{0} \in[0,1]$ and $r_{\alpha}\left(x_{0}\right)$ defined as in (6). Let $\delta>0$. As it is explained in Section 2, one can find sequences $m_{n}^{\prime} \rightarrow+\infty$ and $m_{n} \rightarrow+\infty$ which satisfy (8).

Let $j_{n}=\left[m_{n}\left(r_{\alpha}\left(x_{0}\right)-\delta\right)\right]$. Let $m_{1}^{(n)}$ defined by

$\alpha\left(m_{1}^{(n)}-1\right) \leq j_{n}<\alpha m_{1}^{(n)} \quad$ i.e. $\quad \alpha\left(m_{1}^{(n)}-1\right) \leq m_{n}\left(r_{\alpha}\left(x_{0}\right)-\delta\right)<\alpha m_{1}^{(n)}$.

Let $k_{n}$ such that $\lambda_{j_{n}}\left(x_{0}\right)=\left(j_{n}, k_{n}\right)=\lambda_{n}$.

Recall that $\lambda_{n}^{l}=\left(j_{n}, k_{n}-1\right)$, and $\lambda_{n}^{r}=\left(j_{n}, k_{n}+1\right)$.

As we already mentioned it, we have $d_{j_{n}}\left(x_{0}\right)=\sup \left\{d_{\lambda_{n}^{l}}, d_{\lambda_{n}^{r}}, d_{\lambda_{n}}\right\}$.

On the other hand for $\varepsilon>0$ one can find $M$ such that for $m^{\prime} \geq M(9)$ is satisfied.

Let us consider $3 \lambda_{j}\left(x_{0}\right)=\left[\left(k_{j}-1\right) 2^{-j},\left(k_{j}+2\right) 2^{-j}\left[\right.\right.$. Choose $m^{\prime}$ the smallest integer such that $\frac{K_{m^{\prime}}}{2^{m^{\prime}}}=\frac{k_{m-1}}{2^{m-1}}-\frac{1}{2^{\alpha m}}$ or $\frac{K_{m^{\prime}}}{2^{m^{\prime}}}=\frac{k_{m-1}}{2^{m-1}}+\frac{1}{2^{\alpha m}}$ belongs to $3 \lambda_{j}\left(x_{0}\right)$.

Remark also that it is always possible to choose $j$ large enough such that $m^{\prime} \geq M$.

Thus

$$
\left|\frac{K_{m^{\prime}}}{2^{m^{\prime}}}-x_{0}\right|>2^{-m\left(r\left(x_{0}\right)+\varepsilon\right)}
$$


Since $K_{m^{\prime}} 2^{-m^{\prime}} \in 3 \lambda_{j}\left(x_{0}\right)$ we have

$$
\begin{aligned}
\frac{3}{2^{j+1}} & >2^{-m\left(r\left(x_{0}\right)+\varepsilon\right)} \\
\frac{\ln (3)}{\ln (2)}-1+m\left(r\left(x_{0}\right)+\varepsilon\right) & >j .
\end{aligned}
$$

Thus $j \leq m\left(r_{\alpha}\left(x_{0}\right)+\varepsilon\right)$.

Again define $m_{1}$ such that

$$
\alpha\left(m_{1}-1\right) \leq j<\alpha m_{1} .
$$

We consider the following cases

(a) $r_{\alpha}\left(x_{0}\right) \leq \alpha$.

Thus we have immediately $m_{n} \geq m_{1}^{(n)}$. Since (8) is satisfied, $\frac{K_{m_{n}^{\prime}}}{2^{m_{n}^{\prime}}} \in$ $3 \lambda_{j_{n}}\left(x_{0}\right)$.

It is related to Case 1 and yields

$$
d_{j_{n}}\left(x_{0}\right) \geq C 2^{-\alpha \gamma m_{n}}
$$

Thus for any $\delta>0$

$$
\begin{aligned}
h_{f}\left(x_{0}\right) & =\liminf _{j \rightarrow \infty} \frac{\log d_{\lambda_{j}\left(x_{0}\right)}}{\log 2^{-j}} \\
& \leq \liminf _{n \rightarrow \infty} \frac{\log d_{\lambda_{j_{n}}\left(x_{0}\right)}}{\log 2^{-j_{n}}} \\
& \leq \frac{\alpha \gamma m_{n}}{\left(r_{\alpha}\left(x_{0}\right)-\delta\right) m_{n}}=\frac{\alpha \gamma}{r_{\alpha}\left(x_{0}\right)-\delta} .
\end{aligned}
$$

On the other hand we choose $\varepsilon>0$ small enough so that $r_{\alpha}\left(x_{0}\right)+\varepsilon<$ $\alpha$ if $r_{\alpha}\left(x_{0}\right)<\alpha$, and we will have $r_{\alpha}\left(x_{0}\right)+\varepsilon>\alpha$ if $r_{\alpha}\left(x_{0}\right)=\alpha$. 
i. If $r_{\alpha}\left(x_{0}\right)<\alpha$ then $\alpha\left(m_{1}-1\right) \leq m\left(r\left(x_{0}\right)+\varepsilon\right)<\alpha m$ and $m_{1} \leq m$.

This is again related to Case 1 and yields

$$
d_{j}\left(x_{0}\right) \leq 2^{-\alpha \gamma m} .
$$

Thus we get

$$
h_{f}\left(x_{0}\right) \geq \frac{\alpha \gamma}{r_{\alpha}\left(x_{0}\right)} .
$$

Thus together with $(24)$ we have $h_{f}\left(x_{0}\right)=\frac{\alpha \gamma}{r_{\alpha}\left(x_{0}\right)}$.

Furthermore following the same proof and using the $p$ leader computed in Case ?? we have $u_{f}^{p}\left(x_{0}\right)=\frac{\alpha\left(\gamma+\frac{1}{p}\right)}{r_{\alpha}\left(x_{0}\right)}-\frac{1}{p}$.

ii. If $r\left(x_{0}\right)=\alpha$, then we may have $m \leq m_{1}$, with $\frac{K_{m^{\prime}}}{2^{m^{\prime}}}=\frac{k}{2^{m-1}}+\frac{1}{2^{\alpha m}}$ inside $3 \lambda_{j}\left(x_{0}\right)$.

This yields in all cases again

$$
d_{j}\left(x_{0}\right) \leq 2^{-\alpha \gamma m} .
$$

Thus we get

$$
\begin{aligned}
\frac{\ln \left(d_{j}\left(x_{0}\right)\right)}{\ln \left(2^{-j}\right)} & \geq \frac{\alpha \gamma(m+1)}{j} \\
& \geq \frac{\alpha \gamma m}{m(\alpha+\varepsilon)} \\
& \geq \frac{\alpha \gamma}{\alpha+\varepsilon} .
\end{aligned}
$$

Thus together with (24) we have $h_{f}\left(x_{0}\right)=\frac{\alpha \gamma}{\alpha}=\frac{\alpha \gamma}{r_{\alpha}\left(x_{0}\right)}$. 
The same computation yields $u_{f}^{p}\left(x_{0}\right)=\frac{\alpha\left(\gamma+\frac{1}{p}\right)}{r_{\alpha}\left(x_{0}\right)}-\frac{1}{p}$.

(b) Suppose $r\left(x_{0}\right)>\alpha$. Thus $m_{1}^{(n)}>m_{n}$. It is related to Case 2 and yields

$$
d_{j_{n}}\left(x_{0}\right) \geq C 2^{-\alpha \gamma j_{n}}
$$

Thus we have the upper-bound

$$
\begin{aligned}
h_{f}\left(x_{0}\right) & \leq \liminf _{n \rightarrow \infty} \frac{\log d_{\lambda_{j_{n}}\left(x_{0}\right)}}{\log 2^{-j_{n}}} \\
& \leq \frac{\alpha \gamma j_{n}}{j_{n}}=\alpha \gamma .
\end{aligned}
$$

For the lower bound we pick up $\varepsilon$ small enough and have $r_{\alpha}\left(x_{0}\right)+\varepsilon>$ $\alpha$. This yields that we may have $m \leq m_{1}$ and get

$$
d_{j}\left(x_{0}\right) \leq C 2^{-\alpha \gamma j} .
$$

This yields

$$
. h_{f}\left(x_{0}\right) \leq \alpha \gamma
$$

Together with (30) we have

$$
h_{f}\left(x_{0}\right)=\alpha \gamma
$$

The same computation yields $u_{f}^{p}\left(x_{0}\right)=\alpha\left(\gamma+\frac{1}{p}\right)-\frac{1}{p}$.

\subsubsection{Case $\beta>1$}

Wavelet and $p$-leaders. The coefficients appear every $\alpha \beta$ scales. There is a difference between the scale at which the location of the coefficient appears 
$(\alpha m)$ and the scale at which the coefficient really appears $(\alpha \beta(m-1))$. This is the reason why we need to define these two coefficients $m_{0}$ and $m_{1}$, which satisfy:

$$
\begin{gathered}
\alpha \beta\left(m_{0}-1\right)<j \leq \alpha \beta m_{0}, \\
\alpha\left(m_{1}-1\right) \leq j<\alpha m_{1} .
\end{gathered}
$$

When $\beta>1$, we have the following situation:

0 . Suppose that $\frac{k}{2^{j}}=\frac{K}{2^{m-1}} \pm \frac{1}{2^{\alpha m}}$, with $m \geq m_{0}$. Since we need to have $m \alpha \leq j$, this yields $m_{0} \leq m \leq m_{1}-1$.

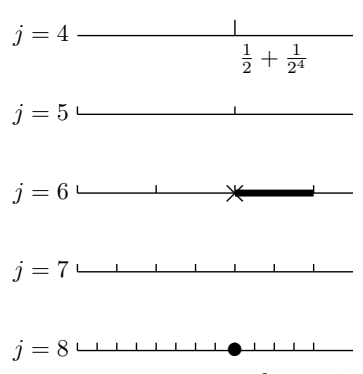

Here we consider an example where $\frac{k}{2^{6}}=\frac{1}{2}+\frac{1}{2^{4}}$. So, with $m=2$, the location of the coefficient appears at scale $\alpha m=4$ and the coefficient appears at scale $\alpha \beta m=8$. Furthermore, since $j=6$, we have $m_{0}=2$

$$
\text { and } m_{1}=4 \text { and then } m_{0} \leq m \leq m_{1}-1 \text {. }
$$

Thus $d_{\lambda}=2^{-\alpha \beta \gamma m}$.

For what concerns the $p$-leader, since $m \leq m_{1}-1$, we can have a non vanishing coefficient located at $\lambda^{\prime} \subset \lambda$ with $\lambda^{\prime}=\left(j^{\prime}, k^{\prime}\right)$ such that $\frac{k^{\prime}}{2^{j^{\prime}}}=\frac{k}{2^{j}}+\frac{1}{2^{\alpha(\alpha m+1)}}$ if $\alpha(\alpha m+1) \geq j$.

Remark that if we set $a_{l}=\alpha^{l} m+\sum_{n=0}^{l-1} \alpha^{n}$ for $l \geq 1$, whenever $\alpha a_{l} \geq j$, we will have a non vanishing coefficient located at $\lambda^{\prime} \subset \lambda$ with $\lambda^{\prime}=\left(j^{\prime}, k^{\prime}\right)$ 
such that $\frac{k^{\prime}}{2^{j^{\prime}}}=\frac{k}{2^{j}}+\sum_{n=1}^{l} \frac{1}{2^{\alpha_{n}}}$. The contribution of these coefficients is anyway at most

$$
\begin{aligned}
\sum_{n=1}^{+\infty} 2^{-a p \alpha \beta a_{n}} & \leq \sum_{n=1}^{+\infty} 2^{-a p \alpha \beta \alpha^{n} m} \leq \sum_{n=1}^{+\infty} 2^{-a p \alpha \beta n \alpha m} \\
& \leq \frac{2^{-a p \alpha^{2} \beta m}}{1-2^{-a p \alpha^{2} \beta m}} \leq \frac{1}{2^{a p \alpha^{2} \beta m}-1}
\end{aligned}
$$

Remark that $\frac{1}{2^{a p \alpha^{2} \beta m}-1} \leq 2^{-a p \alpha \beta m}$ with $C=\frac{1}{2^{a p\left(\alpha^{2}-\alpha\right) \beta}-1}$ independant of $m$.

Otherwise the first scale $j^{\prime}>j$ at which a non vanishing coefficient can appear is $\alpha \beta(j+1)$. This yields in all the cases

$$
\begin{aligned}
2^{-a p \alpha \beta m}+\sum_{\ell=j+1}^{\infty} 2^{\ell-j} 2^{-a p \alpha \beta \ell} & \leq D_{\lambda, p}^{p} \leq(C+1) \times 2^{-a p \alpha \beta m}+\sum_{\ell=j+1}^{\infty} 2^{\ell-j} 2^{-a p \alpha \beta \ell} \\
2^{-a p \alpha \beta m} & \leq D_{\lambda, p}^{p} \leq\left(C+1+\frac{2}{1-2^{-a p \alpha \beta}}\right) 2^{-a p \alpha \beta m} .
\end{aligned}
$$

The other cases are very similar to those already studied with $\beta=1$ (cases 1. and 2. in Section 5.4.1), and we find:

1. suppose that $\frac{k}{2^{j}}=\frac{K}{2^{m^{\prime}}}$ is an irreducible fraction with $m^{\prime} \geq m_{1}$. Then $d_{\lambda}=2^{-\alpha \beta \gamma m^{\prime}}$ and the $p$-leader is bounded by

$$
2^{-a p \alpha \beta m^{\prime}} \leq D_{\lambda, p}^{p} \leq 2^{-a p \alpha \beta m^{\prime}}+C 2^{-a p \alpha \beta j}
$$

2. Suppose $\frac{k}{2^{j}}=\frac{K}{2^{m^{\prime}}}$ (or $\left.\frac{K}{2^{m^{\prime}}}-\frac{1}{2^{j}}\right)$ is an irreducible fraction which is not of the type studied in 0 , with $m^{\prime}<m_{1}$. Thus we will have $d_{\lambda}=2^{-\alpha \beta \gamma(j+1)}$, and

$$
D_{\lambda, p}^{p}=C 2^{-a p \alpha \beta(j+1)}
$$


Computation of the local regularity of $f$ The only case that really differs from what we have done when $\beta=1$ is the case $\alpha<r\left(x_{0}\right) \leq \alpha \beta$. This yields $m_{0}^{(n)} \leq m_{n}<m_{1}^{(n)}$.

It is related to Case 0 . and yields

$$
d_{j_{n}}\left(x_{0}\right) \geq C 2^{-\alpha \beta \gamma m_{n}}
$$

Thus we get

$$
h_{f}\left(x_{0}\right) \leq \frac{\alpha \beta \gamma}{r_{\alpha}\left(x_{0}\right)}
$$

The same computation yields in the case of the $p$ exponent

$$
u_{f}^{p}\left(x_{0}\right) \leq \frac{\alpha \beta\left(\gamma+\frac{1}{p}\right)}{r_{\alpha}\left(x_{0}\right)}-\frac{1}{p} .
$$

On the other hand we have to consider the following cases.

1. If $\alpha<r_{\alpha}\left(x_{0}\right)<\alpha \beta$, choosing $\varepsilon$ small enough we have $\alpha<r_{\alpha}\left(x_{0}\right)+\varepsilon$ and $r_{\alpha}\left(x_{0}\right)+\varepsilon<\alpha \beta$. Using the same notations as in the general setting of this section we get $m_{0} \leq m$ but may have $m<m_{1}$. This is again related to Case 0. and yields

$$
d_{j}\left(x_{0}\right) \leq C 2^{-\alpha \beta \gamma m} .
$$

Thus the following upper-bound holds

$$
h_{f}\left(x_{0}\right) \geq \frac{\alpha \beta \gamma}{r_{\alpha}\left(x_{0}\right)} .
$$

Together with (37) this yields

$$
h_{f}\left(x_{0}\right)=\frac{\alpha \beta \gamma}{r_{\alpha}\left(x_{0}\right)}
$$


The same computation yields $u_{f}^{p}\left(x_{0}\right)=\frac{\alpha \beta\left(\gamma+\frac{1}{p}\right)}{r_{\alpha}\left(x_{0}\right)}-\frac{1}{p}$.

2. If $r_{\alpha}\left(x_{0}\right)=\alpha \beta$ remark that the upperbound (37) yields

$$
h_{f}\left(x_{0}\right) \leq \gamma
$$

Since we already know that $h_{f}\left(x_{0}\right) \geq \gamma$ because $f \in C^{\gamma}(\mathbb{R})$ this yields

$$
h_{f}\left(x_{0}\right)=\frac{\alpha \beta \gamma}{r_{\alpha}\left(x_{0}\right)}=\gamma
$$

For what concerns the $p$ exponent the bound (38) yields

$$
u_{f}^{p}\left(x_{0}\right) \leq \gamma
$$

Since we know already that $u_{f}^{p}\left(x_{0}\right) \geq h_{f}\left(x_{0}\right)=\gamma($ see (18)) we get

$$
u_{f}^{p}\left(x_{0}\right)=\frac{\alpha \beta\left(\gamma+\frac{1}{p}\right)}{r_{\alpha}\left(x_{0}\right)}-\frac{1}{p}=\gamma
$$

This proves Theorem 1.

\subsubsection{Oscillating singularities}

Let $s>0$.

Remark that computing $h_{f}^{s}\left(x_{0}\right)$ or $h_{f}\left(x_{0}\right)$ necessitates the same amount of efforts.

Indeed we have $f^{s}=\sum_{\lambda \in \Lambda(\alpha, \beta)} c_{\lambda}^{s} \psi_{\lambda}^{s}(x)$ where $\psi^{s}$ is the fractional integrate of $\psi$, and $c_{\lambda}^{s}=2^{-j\left(\gamma+s+\frac{1}{2}\right)}$ if $\lambda \in \Lambda(\alpha, \beta)$ and 0 otherwise. 
One could argue that $\left\{\psi_{\lambda}^{s}=2^{j / 2} \psi^{s}\left(2^{j} .-k\right), j \geq 0, k \in \mathbb{Z}\right\}$ is not exactly a wavelet basis. Following [1] we can anyway compute the regularity of $f^{s}$ taking $c_{\lambda}^{s}$ as the amplitude of the coefficients of $f^{s}$ in the formula of the wavelet leaders. This means, following Proposition 2, that we have $h_{f}^{s}\left(x_{0}\right)=\liminf _{j \rightarrow \infty} \frac{\ln \left(\tilde{d}_{j}^{s}\left(x_{0}\right)\right)}{\ln \left(2^{-j}\right)}$ with $\tilde{d}_{j}^{s}\left(x_{0}\right)=\sup _{\lambda^{\prime} \subset 3 \lambda_{j}\left(x_{0}\right)} 2^{j^{\prime} / 2}\left|c_{\lambda^{\prime}}^{s}\right|$.

The location of the non vanishing coefficients is the same in $f^{s}$ and $f$. Their amplitude at scale $j$ is respectively $2^{-j\left(\gamma+s+\frac{1}{2}\right)}$ and $2^{-j\left(\gamma+\frac{1}{2}\right)}$. Thus we can estimate $\tilde{d}_{j}^{s}\left(x_{0}\right)$ with the same formula which yields $d_{j}\left(x_{0}\right)$ taking $\gamma+s$ instead of $\gamma$. This amounts to use the results of Theorem 1 taking $\gamma+s$ instead of $\gamma$. Thus

- if $r_{\alpha}\left(x_{0}\right) \leq \alpha \beta$ we have $h_{f}^{s}\left(x_{0}\right)=\frac{\alpha \beta(\gamma+s)}{r_{\alpha}\left(x_{0}\right)}$,

- if $r_{\alpha}\left(x_{0}\right)>\alpha \beta$ we have $h_{f}^{s}\left(x_{0}\right)=\alpha \beta(\gamma+s)$.

The oscillating exponent follows by Formula (10).

\section{$6 \quad$ Spectra of singularities}

Let $F_{r}^{\alpha}=\left\{x_{0}: r_{\alpha}\left(x_{0}\right) \geq r, r<\infty\right\}$ and $G_{r}^{\alpha}=\left\{x_{0}: r_{\alpha}\left(x_{0}\right)=r, r<\infty\right\}$.

We want to compute the Hausdorff dimension of $G_{r}^{\alpha}$. To do this we will first compute the Hausdorff dimension of $F_{r}^{\alpha}$. We will prove that its Hausdorff dimension is exactly $\frac{1}{r}$.

Remark that the upper bound of the Hausdorff dimension of a set is in general rather straightforward if one can find an appropriate covering of the set. Generally it is more difficult to obtain a lower bound. 
Several results could be used to derive this lower bound but we will make use of recent results by Durand [6] in the version proposed by A. Amou and Y. Bugeaud [3], since this result can be applied directly in our case.

Definition 7. Let $U$ be a real open interval. Let $\left(x_{i}\right)_{i \geq 1}$ be points in $U$ and let $\left(r_{i}\right)_{i \geq 1}$ be a sequence of positive real numbers such that $\lim _{i \rightarrow \infty} r_{i}=0$. The family $\left(x_{i}, r_{i}\right)_{i \geq 1}$ is a homogeneous ubiquitous system in $U$ if the set $\limsup _{i} B\left(x_{i}, r_{i}\right)$ is of full Lebesgue measure in $U$.

We need also a classical extension of the definition of Hausdorff measure.

Definition 8. Let $\mathbf{w}: \mathbb{R}^{+} \rightarrow \mathbb{R}^{+}$be a continuous increasing function satisfying $\mathbf{w}(0)=0$, and let $A$ be a bounded subset of $\mathbb{R}$.

If $|B|$ denotes the diameter of the set $B$, let

$$
M_{\varepsilon}^{\mathbf{w}}(A)=\inf _{R}\left\{\sum_{\left(A_{i}\right) \in R} \mathbf{w}\left(\left|A_{i}\right|\right)\right\}
$$

where the infimum is taken on all coverings $R$ by families of balls $\left(A_{i}\right)_{i \in \mathbb{N}}$ of radius at most $\varepsilon$.

The mes $_{\mathbf{w}}$-measure of $A$ is defined as

$$
\operatorname{mes}_{\mathbf{w}}(A)=\lim _{\varepsilon \rightarrow 0} M_{\varepsilon}^{\mathbf{w}}(A)
$$

For $a>0$ we will make use of the functions $\mathbf{w}_{\mathbf{a}}(x)=|x|^{a}|\log (x)|$.

It is easy to prove that if a set has a $m e s_{\mathbf{w}_{\mathbf{a}}}$-measure strictly positive then its Hausdorff dimension is at most $a$.

Theorem D of [3] proved in [6] yields the following. 
Theorem 7. Let $\tau$ be a real number with $\tau \geq 1$. Let the family $\left(x_{i}, r_{i}\right)_{i \geq 1}$ be a homogeneous ubiquitous system in some open interval $U$.

The Hausdorff dimension of the set $\lim \sup B\left(x_{i}, r_{i}^{\tau}\right)$ is at least equal to $\frac{1}{\tau}$. Furthermore $\operatorname{mes}_{\mathbf{w}_{\frac{1}{\tau}}}\left(\lim \sup B\left(x_{i}, r_{i}^{\tau}\right)\right)=+\infty$.

Our goal is to prove the following Lemma.

Lemma 1. Let $r \geq 1$ and $F_{r}^{\alpha}=\left\{x_{0}: r_{\alpha}\left(x_{0}\right) \geq r, r<\infty\right\}$ and $G_{r}^{\alpha}=\left\{x_{0}:\right.$ $\left.r_{\alpha}\left(x_{0}\right)=r, r<\infty\right\}$. Then

1. The Hausdorff dimension of $F_{r}^{\alpha}$ is exactly $\frac{1}{r}$.

2. The Hausdorff dimension of $G_{r}^{\alpha}$ is exactly $\frac{1}{r}$.

Proof. 1. We first build a homogeneous ubiquitous system $\left(x_{m}, r_{m}\right)_{m \geq 1}$ such that $\limsup _{m \rightarrow+\infty} B\left(x_{m}, r_{m}^{r}\right) \subset F_{r}^{\alpha}$.

We want to have $\mathcal{S}_{\alpha}=\left\{x_{m}, m \in \mathbb{N}\right\}$. Since the points of $\mathcal{S}_{\alpha}$ are indexed by $k$ and $j$ we need to reindex it.

Let $x_{2 n}=\frac{k}{2^{j-1}}-\frac{1}{2^{\alpha j}}$ and $x_{2 n+1}=\frac{k}{2^{j-1}}+\frac{1}{2^{\alpha j}}$ with $0 \leq k \leq 2^{j}-1$ and $n=k+2^{j-1}$. We set $r_{2 n}=r_{2 n+1}=2^{-(j-1)}$ for $2^{j-1} \leq n \leq 2^{j}-1$.

Following (7) $\lim \sup B\left(x_{m}, r_{m}\right)$ is of full Lebesgue measure. Thus following Definition 7 the set $\left(x_{i}, r_{i}\right)_{i \geq 1}$ is a homogeneous ubiquitous system.

We have

$$
\limsup _{m \rightarrow+\infty} B\left(x_{m}, r_{m}^{r}\right) \subset F_{r}^{\alpha}
$$


with $x_{m} \in S_{\alpha}, x_{m}=\frac{k_{j_{m}-1}}{2^{j_{m}-1}}+\frac{\varepsilon_{j_{m}}}{2^{\alpha j_{m}}}$ and $\varepsilon_{j_{m}} \in\{1,-1\}$.

Indeed, suppose $x_{0} \in \limsup B\left(x_{m}, r_{m}^{r}\right)$. For all $m \in \mathbb{N}$, there exists $n \geq m$ such that $x_{0} \in B\left(x_{n}, r_{n}^{r}\right)$, so

$$
\begin{aligned}
&\left|x_{n}-x_{0}\right| \leq 2^{-r \times r_{n}} \\
& \frac{\log \left|\frac{k_{j_{n}-1}}{2^{j_{n}-1}}+\frac{\varepsilon_{j}}{2^{\alpha j_{n}}}-x_{0}\right|}{\log \left(2^{j_{n}-1}\right)} \geq r,
\end{aligned}
$$

Remark that we have

$$
r_{\alpha}\left(x_{0}\right)=\limsup _{n \rightarrow+\infty} \frac{\log \left|\frac{k_{j_{n}-1}}{2^{j_{n}-1}}+\frac{\varepsilon_{j}}{2^{\alpha j_{n}}}-x_{0}\right|}{\log \left(2^{j_{n}}\right)}=\limsup _{n \rightarrow+\infty} \frac{\log \left|\frac{k_{j_{n}-1}}{2^{j_{n}-1}}+\frac{\varepsilon_{j}}{2^{\alpha j_{n}}}-x_{0}\right|}{\log \left(2^{j_{n}-1}\right)}
$$

Thus we have $r_{\alpha}\left(x_{0}\right) \geq r$. Hence the first inclusion.

Furthermore let $\delta>0$ such that $r-\delta>0$. We have in fact

$$
\limsup _{m \rightarrow+\infty} B\left(x_{m}, r_{m}^{r}\right) \subset F_{r}^{\alpha} \subset \limsup _{m \rightarrow+\infty} B\left(x_{m}, r_{m}^{r-\delta}\right)
$$

Indeed we can use (8) and (44) on the right-hand side inequality and the conclusion is straightforward.

Denote $A_{r}=\limsup _{m \rightarrow+\infty} B\left(x_{m}, r_{m}^{r}\right)$ for $r \geq 1$

We have two cases.

(a) Suppose $r=1$. 
Since $A_{1}=\limsup _{m \rightarrow+\infty} B\left(x_{m}, r_{m}\right)$ is of full Lebesgue measure, we have clearly $\operatorname{dim}\left(F_{\alpha}^{1}\right)=1$.

(b) Suppose $r>1$.

- We start by computing an upper bound for the Hausdorff dimension of $F_{r}^{\alpha}$.

Take $\delta^{\prime}$ and $\delta$ such that $\delta^{\prime}>\delta$ and $r-\delta>r-\delta^{\prime}>1$. We can compute an upper-bound for the $\frac{1}{r-\delta^{\prime}}$ dimensional Hausdorff measure of $A_{r-\delta}$. Indeed by definition $A_{r-\delta}=\bigcap_{n \in \mathbb{N}} \bigcup_{m \geq n} B\left(x_{m}, r_{m}^{r-\delta}\right)$. Thus for all $n \in \mathbb{N}$

$$
\begin{aligned}
\operatorname{mes}_{\frac{1}{r-\delta^{\prime}}}\left(A_{r-\delta}\right) & =m e s_{\frac{1}{r-\delta^{\prime}}}\left(\bigcap_{n \in \mathbb{N}} \bigcup_{m \geq n} B\left(x_{m}, r_{m}^{r-\delta}\right)\right) \\
& \leq \sum_{m=0}^{\infty} m e s_{\frac{1}{r-\delta^{\prime}}}\left(B\left(x_{m}, r_{m}^{r-\delta}\right)\right. \\
& \leq \sum_{j=0}^{\infty} 2^{j} 2^{-(j-1)\left(\frac{r-\delta}{r-\delta^{\prime}}\right)} \\
& \leq \sum_{j=1}^{\infty} 2^{-j\left(\frac{r-\delta}{r-\delta^{\prime}}-1\right)}=C .
\end{aligned}
$$

Thus for all $\delta^{\prime}>\delta$ we have mes $\frac{1}{r-\delta^{\prime}}\left(A_{r-\delta}\right) \leq C$, which proves $\operatorname{dim}\left(A_{r-\delta}\right) \leq \frac{1}{r-\delta}$.

Thus for all $\delta>0$ we have $\operatorname{dim}\left(F_{r}^{\alpha}\right) \leq \frac{1}{r-\delta}$. This proves $\operatorname{dim}\left(F_{r}^{\alpha}\right) \leq \frac{1}{r}$.

- Let now give a lower bound for the Hausdorff dimension of $F_{r}^{\alpha}$.

Since $\left(x_{m}, r_{m}\right)_{m \geq 1}$ is a homogeneous ubiquitous system, we can 
apply Theorem 7 and get a lower bound for $\operatorname{dim}\left(A_{r}\right)$. This yields immediately that $\operatorname{dim}\left(F_{r}^{\alpha}\right) \geq \frac{1}{r}$.

Combining the upper bound and the lower bound we get $\operatorname{dim}\left(F_{r}^{\alpha}\right)=$ $\frac{1}{r}$.

2. Let compute the Hausdorff dimension of $G_{r}^{\alpha}$.

Remark that $F_{r}^{\alpha}=G_{r}^{\alpha} \bigcup_{n \in \mathbb{N}^{\star}} F_{r+\frac{1}{n}}^{\alpha}$. Thus we have $\operatorname{dim}\left(G_{r}^{\alpha}\right) \leq \frac{1}{r}$.

Following Theorem 7 we have $\operatorname{mes}_{\mathbf{w}_{\frac{1}{\mathrm{r}}}}\left(F_{r}^{\alpha}\right)=+\infty$.

On the other hand since $\operatorname{dim}\left(F_{r+\frac{1}{n}}\right)=\frac{1}{r+\frac{1}{n}}$, using standart computations (see for example Chapter 2.5 of [7]) mes $_{\mathbf{w}_{\frac{1}{\mathrm{r}}}}\left(F_{r+\frac{1}{n}}^{\alpha}\right)=0$.

Since $F_{r}^{\alpha}=G_{r}^{\alpha} \bigcup_{n \in \mathbb{N}^{\star}} F_{r+\frac{1}{n}}^{\alpha}$ we have $\operatorname{mes}_{\mathbf{w}_{\frac{1}{\mathbf{r}}}}\left(G_{r}^{\alpha}\right)=+\infty$.

Thus $\operatorname{dim}\left(G_{r}^{\alpha}\right) \geq \frac{1}{r}$, which yields the result.

By Lemma 1 we get immediately Corollary 2 and Corollary 3.

\section{Multifractal formalism}

Let us now check if the function $f$ satisfies a formula of multifractal formalism type. 


\subsection{Multifractal formalism with Oscillation spaces}

Jaffard in [17] (Definition 15) gives a multifractal type formula to compute the Hölder spectrum of singularities, the so called multifractal formalism for Hölder spectrum. This formula, unlike previous formulas which were stated before, is stable under oscillating behaviors and is easy to compute once we have the wavelet leaders $d_{\lambda}$. We will check that it is satisfied in our case.

Recall the definition with the help of wavelet leaders. Indeed we want to compute the following function of $q$

$$
\omega_{f}(q)=\sup \left\{s: \forall j \geq 0,2^{j(s-1)} \sum_{\lambda \in \Lambda_{j}} d_{\lambda}^{q}<+\infty\right\}
$$

Then the multifractal formalism claims $d(h)=d_{O}(h)$ with

$$
d_{O}(h)=\inf _{q}\left(h q-\omega_{f}(q)+1\right) .
$$

Let us check if this formula is true for our function $f$.

As usual define $m_{0}=\left\lceil\frac{j}{\alpha \beta}\right\rceil$ and $m_{1}=\left\lfloor\frac{j}{\alpha}\right\rfloor$. We have $2^{j}$ dyadics intervals at scale $j$ inside $[0,1]$ with $2^{m}$ irreducible fractions of type $\frac{k}{2^{m-1}} \pm \frac{1}{2^{\alpha m}}$ for $m_{0} \leq m \leq m_{1}$ with a general count of $2^{\alpha m-1}$ of irreducible fractions at scale $\alpha m$.

Let

$$
\Omega_{f}(j, q)=2^{j(s-1)}\left(A_{1}(j, q)+A_{2}(j, q)+A_{3}(j, q)\right),
$$


with

$$
\begin{aligned}
& A_{1}(j, q)=\sum_{m=0}^{m_{1}-1} 2^{m-1} 2^{-q \alpha \gamma \beta(j+1)}, \\
& A_{2}(j, q)=\sum_{m=m_{1}}^{j} 2^{m-1} 2^{-\alpha \beta \gamma q m} \\
& A_{3}(j, q)=\sum_{m=m_{0}}^{m_{1}-1} 2^{m} 2^{-\alpha \beta \gamma q m} .
\end{aligned}
$$

In $\Omega_{f}(j, q)$ we sum on each scale $m$ all the contributions of leaders located at irreducible fractions $\frac{K}{2^{m}}$. Actually irreducible fractions of type $\frac{k}{2^{m^{\prime}-1}} \pm \frac{1}{2^{\alpha m^{\prime}}}$ are counted twice. But we can say that we have anyway

$$
\frac{1}{2} \Omega_{f}(j, q) \leq 2^{j(s-1)} \sum_{\lambda \in \lambda_{j}} d_{\lambda}^{q} \leq \Omega_{f}(j, q)
$$

This yields the following cases

1. Suppose $1-\alpha \gamma \beta q<0$, which is equivalent to $\frac{1}{\alpha \gamma \beta}<q$. We have

$$
\begin{aligned}
& A_{1}(j, q)=2^{-q \alpha \gamma \beta(j+1)} \sum_{m=0}^{m_{1}-1} 2^{m-1} \sim C 2^{-q \alpha \gamma \beta j+\frac{j}{\alpha}} \quad \sim C 2^{m_{1}} 2^{-\alpha \beta \gamma q m_{1}}=C 2^{\frac{j}{\alpha}-q \beta \gamma j}, \\
& A_{2}(j, q) \quad \sim C 2^{m_{0}} 2^{-\alpha \beta \gamma q m_{0}}=C 2^{\frac{j}{\alpha \beta}-q \gamma j} . \\
& A_{3}(j, q) \quad
\end{aligned}
$$

We have clearly $A_{1}(j, q)<<A_{2}(j, q)$.

Let compare $A_{2}(j, q)$ and $A_{3}(j, q)$. We have $\frac{1}{\alpha}-q \beta \gamma=\beta\left(\frac{1}{\alpha \beta}-q \alpha \beta \gamma\right) \leq$ $\frac{1}{\alpha \beta}-q \alpha \beta \gamma$ since $\frac{1}{\alpha \beta}-q \alpha \beta \gamma<0$ and $\beta \geq 1$.

Thus if $\beta=1$ we have $A_{2}(j, q) \sim A_{3}(j, q)$ and if $\beta>1 A_{3}(j, q)>>A_{2}(j, q)$. 
Thus in all cases we have

$$
\Omega_{f}(j, q) \sim C 2^{j\left(s-1+\frac{1}{\alpha \beta}-q \gamma\right)} .
$$

This yields, following $(47) \omega_{f}(q)=-\frac{1}{\alpha \beta}+q \gamma+1$.

2. Suppose $1-\alpha \gamma \beta q \geq 0$, thus $\frac{1}{\alpha \gamma \beta} \geq q$ then we have, following the same method as previously

$$
\begin{gathered}
A_{1}(j, q)=2^{-q \alpha \gamma \beta(j+1)} \sum_{m=0}^{m_{1}-1} 2^{m-1} \sim C 2^{-q \alpha \gamma \beta j+\frac{j}{\alpha}}, \\
A_{2}(j, q) \quad \sim C 2^{j} 2^{-\alpha \beta \gamma q j}=C 2^{j(1-\alpha \beta \gamma q)}, \\
A_{3}(j, q) \quad \sim C 2^{m_{1}} 2^{-\alpha \beta \gamma q m_{1}}=C 2^{\frac{j}{\alpha}-q \beta \gamma j} .
\end{gathered}
$$

Remark that $1-\alpha \beta \gamma q=\alpha\left(\frac{1}{\alpha}-q \beta \gamma\right)$. Since $\alpha \geq 1$ and $1-\alpha \beta \gamma q \geq 0$ we have $A_{2}(j, q) \sim A_{3}(j, q)$ if $\alpha \geq 1$ and $A_{2}(j, q)>>A_{3}(j, q)$ if $\alpha>1$.

Once again we have

$$
\Omega_{f}(j, q) \sim C 2^{j(s-1+1-\alpha \beta \gamma q)} .
$$

This yields $\omega_{f}(q)=q \alpha \beta \gamma$.

Let $h$ be fixed and $f(q)=h q-\omega_{f}(q)+1$. We have

$$
f(q)=\left\{\begin{array}{cc}
h q-\gamma q+\frac{1}{\alpha \beta} & \text { if } \frac{1}{\gamma \beta \alpha}<q, \\
h q-\alpha \beta \gamma q+1 & \text { otherwise. }
\end{array}\right.
$$

- Suppose $h>\alpha \gamma \beta$ or $h<\gamma$. Thus $f$ is unbounded from below and $d_{O}(h)=$ $-\infty$

- Suppose $\gamma \leq h \leq \alpha \gamma \beta$. The minimum of $f$ is at $p_{0}=\frac{1}{\alpha \gamma \beta}$ and we have 


$$
f\left(p_{0}\right)=\frac{h}{\alpha \gamma \beta}
$$

Since $d_{f}(h)=d_{O}(h)$ for all $h$ the multifractal formalism with oscillation spaces is satisfied.

\subsection{Multifractal formalism with $p$-Oscillation spaces}

The same kind of formula as in the Hölder case exists in order to compute the $p$ spectrum. The claim is the following (see [14] for details): compute $\omega_{f}(p, q)=\sup \left\{s: \forall j \geq 0,2^{j(s-1)} \sum_{\lambda \in \lambda_{j}} D_{\lambda, p}^{q}<+\infty\right\}$.

Then

$$
d_{p}(h)=\inf _{q}\left(h q-\omega_{f}(p, q)+1\right)
$$

should give $d_{p}(h)=d_{f, p}(h)$.

We can check that this is actually true for our function $f$. Indeed remark that it is enough to replace $\gamma$ by $a$ in the previous computation of Section 7.1 to compute exactly the formula for the $p$ spectrum and get it. The multifractal formalism for the $p$ exponent is satisfied.

Acknowledgments. The authors would like to thank Stéphane Jaffard and the two referees for their insightful and accurate comments, which helped to improve the paper significantly.

\section{References}

[1] Arneodo, A. Bacry, E., Jaffard, S. and Muzy J-F.: Oscillating singularities on Cantor sets: A grandcanonical multifractal formalism.J. Stat. Phys 87, 179-209 (1997) 
[2] Arneodo, A. Bacry, E. and Muzy J-F.: The thermodynamics of fractals revisited with wavelets. Physica A 213, 232-275 (1995)

[3] Amou, M. and Bugeaud, Y.: Exponents of Diophantine approximation and expansions in integer bases. J. London Math. Soc. 81 (2) 297-316 (2010)

[4] Calderón, A. P. and Zygmund A.: Local properties of solutions of elliptic partial differential equations. Studia Matematica 20 171-227 (1961)

[5] Daubechies, I: Ten lectures on wavelets. SIAM: Society for Industrial and Applied Mathematics. (1992)

[6] Durand, A.: Sets with large intersection and ubiquity. Math. Proc. Cambridge Philos. Soc. 144 119-144 (2008)

[7] Falconer, K.: Fractal Geometry: Mathematical Foundations and Applications. Wiley (1990)

[8] Fraysse, A.: Regularity criteria for almost every function in Sobolev spaces. J. Funct. Anal. 258, no. 6, 1806-1821. (2010)

[9] Hernández, E. and Weiss, G.: A First Course on Wavelets. CRC Press, New York, (1996).

[10] Jaffard, S., Abry, P. and Lashermes, B.: Wavelet Leaders in Multifractal Analysis. Wavelet Analysis and Applications Tao Qian, Mang I. Vai and Xu Yuesheng, Eds. Applied and Numerical Harmonic Analysis, 219264, Springer (2006)

[11] Jaffard, S., Abry, P. and Roux, S.: Function spaces vs. scaling functions : Tools for image classification Mathematical Image processing, M. Bergounioux Ed., Springer proceedings in Math. Vol 5, pp. 1-40, (2011) 
[12] Heurteaux, Y. and Jaffard, S.: Multifractal analysis of images: new connexions between analysis and geometry. Proceedings of the NATO-ASI Conference on Imaging for Detection and Identification, Springer (2006)

[13] Jaffard, S. and Melot, C.: Wavelet Analysis of Fractal Boundaries. Part 1: Local Exponents. Commun. Math. Phys. 258 513-539 ( 2005)

[14] Jaffard, S. and Melot, C.: Wavelet Analysis of Fractal Boundaries. Part 2: Multifractal analysis. Commun. Math. Phys. 258 541-565 (2005)

[15] Jaffard, S. and Meyer, Y.: Wavelet methods for pointwise regularity and local oscillation of functions. Mem. Am. Math. Soc. 123 no. 587 (1996)

[16] Jaffard, S.: Construction de fonctions multifractales ayant un spectre de singularités prescrit. C.R. Acad. Sci. Série I. 315, 19-24. (1992)

[17] Jaffard, S.: Wavelet techniques in multifractal analysis, in Fractal Geometry and Applications: A Jubilee of Benoit Mandelbrot, M. Lapidus et M. van Frankenhuijsen Eds., Proceedings of Symposia in Pure Mathematics (AMS) 72 Part 2,91-151 (2004)

[18] Meyer, Y.: Ondelettes et opérateurs, Herman, Paris (1990)

[19] Parisi G. and Frisch U.: On the singularity structure of fully developed turbulence; appendix to Fully developed turbulence and intermittency, by $\mathrm{U}$. Frisch; Proc. Int. Summer school Phys. Enrico Fermi, 84-88 North Holland. (1985)

[20] Wendt H., Abry P., Roux S.G, Jaffard S. and Vedel B.: The contribution of wavelets in multifractal analysis; In Series in contemporary applied mathematics, A. Damlamian, S. Jaffard, L.T. Tsien, Eds., Higher education press and World scientific publishing, (2009) 